\title{
An Ultra-Cheap Light Field Microscope for Volumetric Cellular Imaging
}

This paper was downloaded from TechRxiv (https://www.techrxiv.org).

\section{LICENSE}

CC BY 4.0

SUBMISSION DATE / POSTED DATE

$14-08-2021$ / 25-08-2021

\section{CITATION}

Zheng, Zhiyu; Wu, Yinzhe; Gurung, Amul; Liu, Xinyue; Li, Lai Yan; Zhu, Haobo; et al. (2021): An Ultra-Cheap Light Field Microscope for Volumetric Cellular Imaging. TechRxiv. Preprint. https://doi.org/10.36227/techrxiv.15167478.v1

$\mathrm{DOI}$ 


\section{An Ultra-Cheap Light Field Microscope for Volumetric Cellular Imaging}

Zhiyu Zheng,", Yinzhe Wu^ ${ }^{\wedge}$, , Amul Gurung, Xinyue Liu, Lai Yan Li, Haobo Zhu, Carmel L. Howe, Peter Quicke, Simon R. Schultz

Department of Bioengineering, Imperial College London, London SW7 2AZ, UK

\begin{abstract}
We present a novel light field microscope (LFM) hardware design which benefits from scaled productions of cameras and other optical components. Our design has dramatically lower cost $(<$ GBP 2000) yet only slightly compromised performance.

Clinical Relevance-This novel LFM design enables transient volumetric imaging at a cellular resolution at a much lower cost.
\end{abstract}

\section{INTRODUCTION}

Conventional microscopy techniques (twophoton, confocal microscopy) have high spatial resolution, but are prohibitively slow for real-time volumetric imaging as they require a single point scanned in a volume. For example, a two-photon microscope with an $8 \mathrm{kHz}$ resonant scanner takes over $6 \mathrm{sec}$ to scan a $1.0 \times 1.0 \times 0.1 \mathrm{~mm}$ volume [1], and costs over $£ 100,000$. Light field microscopy (LFM) is a scanless method that has been applied to live brain tissue imaging[3]. It enables high speed volumetric imaging[2]. However, state-of-the-art LFMs cost over $£ 30,000$, limiting the potential application domain. This study demonstrates an ultra-cheap LFM with cellular resolution ( $\sim 10 \mathrm{um})$ and a total cost less than $£ 2000$.

\section{METHODS}

Our LFM follows the conventional configuration developed by Levoy et al. where a microlens array (MLA) is placed at the intermediate image plane of a wide field microscope and the image sensor is at the relay-lens-projected MLA's back focal plane (see Figure $1^{+}$)[2]. Synthetic refocusing and deconvolution were applied to reconstruct 3D volumes from the captured 4D (spatial coordinates Sx, Sy and angular coordinates Ax, Ay) light field [3].

\footnotetext{
Correspondence Authors \{zhiyu.zheng18, yinzhe.wu18, s.schultz\}@imperial.ac.uk

\# Equal Contribution

* Theoretically calculated resolutions

** Actual magnification, due to tube lens focal length being $200 \mathrm{~mm}$ instead of $180 \mathrm{~mm}$.

See Supplementary Materials for more information.
}

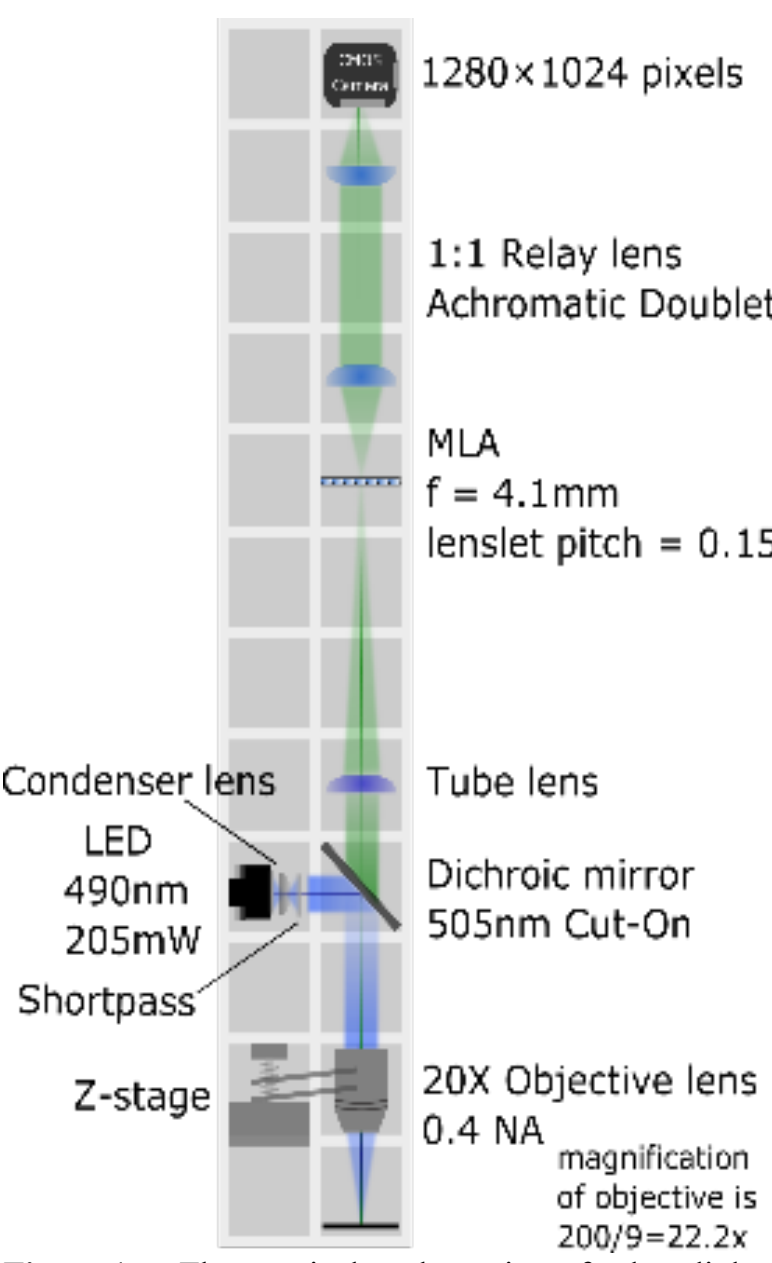

Figure 1. The optical schematic of the light field microscope.

To save cost and enable modularity, our LFM utilizes the UC2 toolbox[4] and replaces expensive metal optomechanical components with a 3Dprinted plastic cube-based framework (see Figure 2). To further reduce the cost, cheaper optical components were chosen while making compromises on spatial resolution, field of view, sensor signal-to-noise ratio, and distortion correction. We shall now elaborate on some key components and image reconstruction methods. 


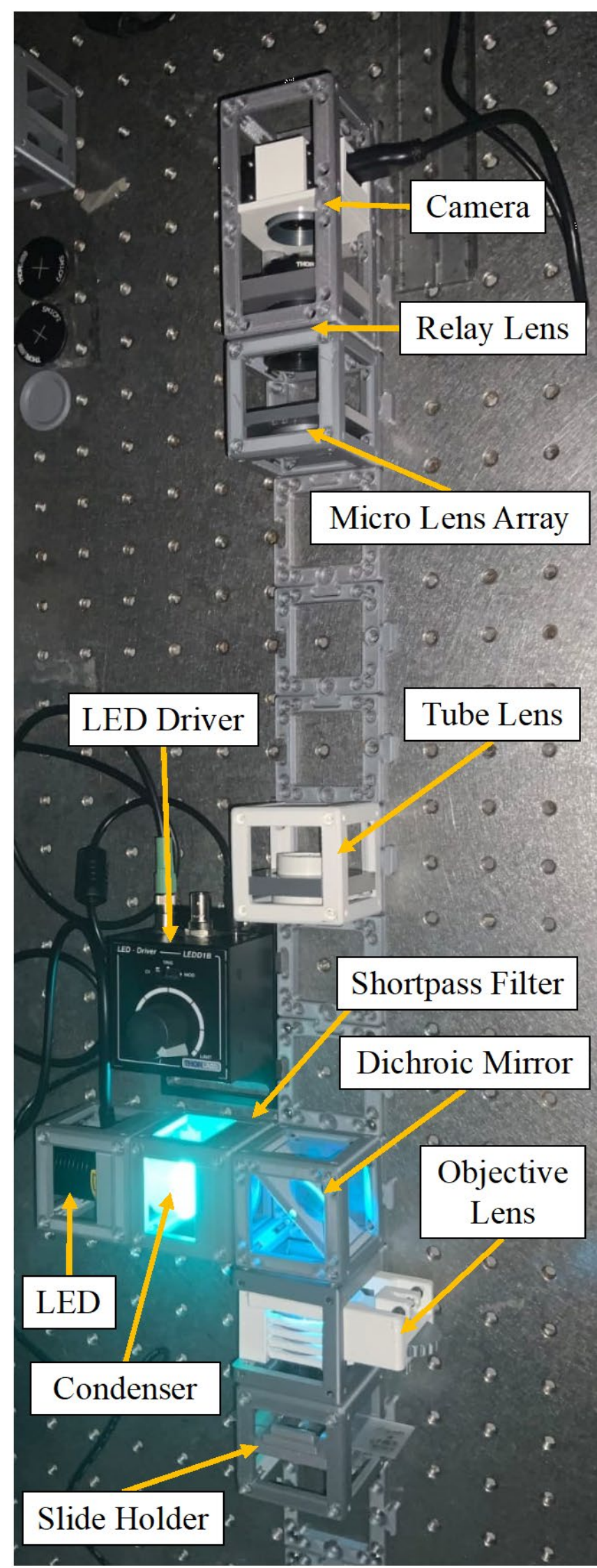

Figure 2. The photo of the light field microscope.
USB Camera: For imaging neuron activities, the number and size of pixel used in [3] is redundant, smaller pixels are sufficient to capture neuron discharges, considering the high latency of fluorescent dyes[3]. Therefore, we used a cheaper CMOS camera with both lower size and smaller number of pixel (FLIR Chameleon 3, 1280×1024 pixels, pixel size $4.8 \mu \mathrm{m})$, which can replace the expensive one (e.g., ORCA Flash 4 V2, 2048×2048 pixels, $6.5 \mu \mathrm{m}$ pixel size, Hamamatsu[3]). In addition, Chameleon 3 camera has a high frame rate $(149 \mathrm{~Hz})$, which enables fast neuron activity imaging.

Microlens array(MLA): To use the camera sensor efficiently and have a larger aperture, an MLA (MLA150-5C(-M), Thorlabs) which has the smallest f-number among all easily available ones was used. The f-number of MLA is calculated to be 27.3 from its focal length $4.1 \mathrm{~mm}$ divided by lenslet pitch $150 \mu \mathrm{m}[5]$. To project in-focus images from the MLA to the image sensor of the camera, an achromatic pair (MAP104040-A, Thorlabs) is used as a relay lens in between.

Objective lens: As we aim for cellular resolutions rather than the sub-cellular, an objective lens with a smaller magnification (20x objective lens, Olympus RMS20X) can be used here, while able to maintain sufficient lateral resolution $(6.75 \mu \mathrm{m})$ for cellular imaging[3].

To match the MLA, a 200mm-focal-length tube lens was used together with the objective, resulting in a $22.2 x$ magnification and an f-number of 27.8. See Supplementary materials for detailed calculations.

A z-stage from UC2[4] was used to enable the objective to be translated vertically at submillimeter steps. This allows live precise positioning of the objective to keep various samples in focus.

LED: To reduce the cost of light source for illuminating samples, the LED in our design constitute 4 parts including mounted LED (M490L4, Thorlabs), Aspheric Condenser Lens (ACL25416U-A, Thorlabs), T-cube LED driver (LEDD1B, Thorlabs) and power supply (KPS101, Thorlabs). We adopted Epi-illumination where a dichroic mirror (DMLP505, Thorlabs) is placed at a $45^{\circ}$ angle and reflects the excitation signal from 
LED towards specimen while transmitting the emission signal towards objective lens. To save costs, a condenser lens (ACL25416U-A, Thorlabs) was used together with an adapted lens tube (SM1V10, Thorlabs, modified for 3D printing) to collimate the LED light.

Synthetic refocusing: treating shifted sub-aperture images, while the shift is proportional to the obliquity of the viewing angle reflected as multiples of angular components $A_{x}$ and $A_{y}$. The refocusing process can be shown by the following formula:

$$
I(x, y)=I\left(S_{x}, S_{y}\right)=\sum_{A_{x}, A_{y}}\left(\mathcal{L}\left(A_{x}, A_{y}, S_{x}+A_{x}(1-1 / \alpha), S_{y}+A_{y}(1-1 / \alpha)\right)\right)
$$

where $I$ is the intensity at the refocused image, and $\alpha$ is the proportion of the refocused depth with respect to the native focal plane[3]. The $x$ and $y$ coordinates in $I$ correspond to $S_{x}$ and $S_{y}$ in the LF domain. Uniform intensity at specific light path is assumed to eliminate the z-component of the LF domain, and thus the number of dimensions can be reduced[2].
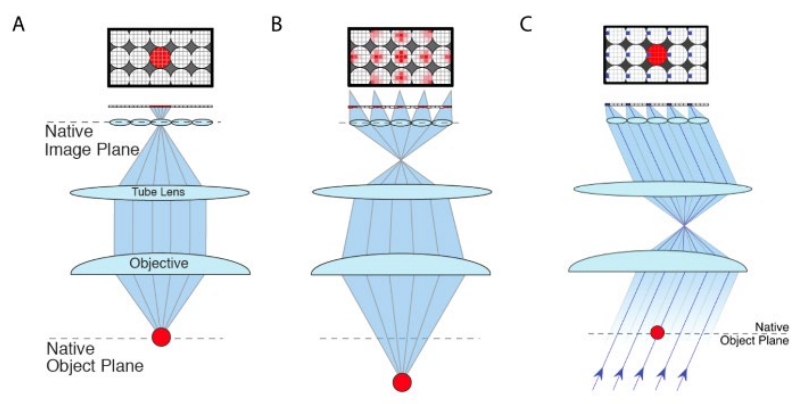

Figure 3. (A) The camera sensor is placed at the back focal plane of the MLA. The LF image contains unique spatial and angular information about sources at (B) different depths and (C) angles of view. Recombining such information is essential for reconstructing the image at different depths from the focal length. Reprinted from Broxton[6]

Deconvolution: an inverse tomographic method that restores the original 3D space from the effect of the filter, point spread function(PSF) that describes the way a microscope collects electric field at a small distance from the focal plane of the MLA, calculated at various depths[4], applying ISRA algorithm to maximise the likelihood function in the 3D volume given distribution of intensity. The ISRA algorithm is denoted as

$$
x^{k+1}(r)=x^{k}(r) \frac{h(-r) * y(r)}{h(-r) * h(r) * x^{k}(r)}
$$

where $x^{k}$ denotes the $3 \mathrm{D}$ reconstruction after $k$-th iteration and convolution with $h(r)$ and $h(-r)$ are equivalent to backward (to the volumetric domain) and forward(to the LF domain) propagation respectively, which allows minimisation of error[7].

\section{RESULTS}

We used the LFM to image green fluorescent protein (GFP) labelled neurons in fixed mouse brain slices, and $0.5 \mu \mathrm{m}$ yellow-green (486nm) fluorescent beads. From the LFM images we successfully implemented both synthetic refocusing and deconvolution and obtained 3D image stacks (see Figure 4). However, in some parts of the images, warping and aberrations exist due to small misalignments and low-quality components. Cost wise, we reduced the cost by an order of magnitude while retaining basic performance and meeting the requirement for cellular resolution imaging (see Table 1 and 2).
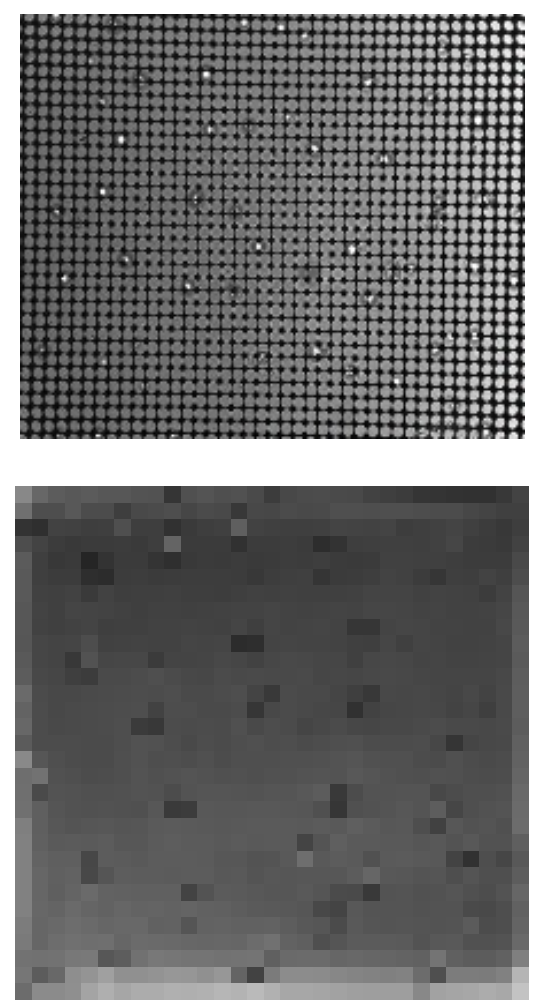

Figure 4. Top: raw LFM image of $0.5 \mu \mathrm{m}$ fluorescent microbeads, Bottom: corresponding ISRA-deconvolved reconstructed volumetric image $(31 \times 31$ pixel, gaze size 214 $\times 214 \mathrm{um}$ ) slice at the native focal plane with 5 iterations. 
TABLE I. COST COMPARISON BETWEEN THE (A) DESIGNED MICROSCOPE ${ }^{+}$AND (B) THE STATE-OF-THE-ART LFM BY QUICKE[3]

\begin{tabular}{|c|c|c|c|c|}
\hline & $\begin{array}{l}\text { (a) Our } \\
\text { microscope }\end{array}$ & & (b) Quicke [3] & \\
\hline & Components & Price & Components & Price \\
\hline Camera & $\begin{array}{l}\text { CMOS: FLIR } \\
\text { Chameleon }{ }^{\circledR} 3 \\
\text { USB3.0 }\end{array}$ & $£ 361.25$ & $\begin{array}{l}\text { sCMOS: } \\
\text { Hamamatsu } \\
\text { ORCA Flash } 4 \\
\text { V2 }\end{array}$ & $\sim £ 15,000$ \\
\hline $\begin{array}{r}\text { Objective } \\
\text { lens } \\
\end{array}$ & $\begin{array}{l}\text { 20X Olympus } \\
\text { Plan Achromat } \\
\text { Objective lens }\end{array}$ & $£ 353.85$ & $\begin{array}{l}25 \mathrm{X} \text { Olympus } \\
\text { Objective }\end{array}$ & $\sim £ 10,000$ \\
\hline MLA & $\begin{array}{l}\text { Thorlabs } \\
\text { MLA150-5C(- } \\
\text { M) }\end{array}$ & $£ 366.84$ & $\begin{array}{l}\text { RPC photonics } \\
\text { MLA-S125-f10 }\end{array}$ & $\sim £ 300$ \\
\hline $\begin{array}{r}\text { Miscellan } \\
\text { eous }^{+} \\
\end{array}$ & & $£ 863.03$ & & $\sim £ 7,250$ \\
\hline Total & & $1,944.97$ & & $\sim £ 32,550$ \\
\hline
\end{tabular}

TABLE II. PARAMETER AND RESOLUTION COMPARISON WITH THE STATE-OF-THE-ART LFM BY QUICKE[3]

\begin{tabular}{|c|c|c|c|}
\hline & & $\begin{array}{l}\text { (a) Our } \\
\text { microscope }\end{array}$ & (b) Quicke \\
\hline \multirow[t]{5}{*}{$\begin{array}{l}\text { Paramet } \\
\text { ers }\end{array}$} & $\begin{array}{l}\text { Objective } \\
\text { magnification }\end{array}$ & $22.2 x^{* *}$ & $25 x$ \\
\hline & Objective $N A$ & 0.4 & 1.0 \\
\hline & Pixel size & $4.8 \mu \mathrm{m}$ & $6.5 \mu \mathrm{m}$ \\
\hline & Pixel per lens & $32 \times 32$ & $45 \times 45$ \\
\hline & Frame rate & $\begin{array}{l}149 \\
\text { frames/sec }\end{array}$ & $\begin{array}{l}100 \\
\text { frames/sec }\end{array}$ \\
\hline \multirow[t]{2}{*}{$\begin{array}{l}\text { Resoluti } \\
\text { ons }{ }^{*}\end{array}$} & $\begin{array}{l}\text { Lateral } \\
\text { resolution }\end{array}$ & $6.75 \mu \mathrm{m}$ & $5 \mu \mathrm{m}$ \\
\hline & $\begin{array}{l}\text { Axial } \\
\text { resolution }^{*}\end{array}$ & $21.18 \mu \mathrm{m}$ & $7.81 \mu \mathrm{m}$ \\
\hline
\end{tabular}

\section{DISCUSSION \& CONCLUSION}

The first version of our ultra-cheap LFM design already functions at cellular spatial resolution and more than $100 \mathrm{~Hz}$ temporal resolution, though with some deficiencies, including (1) compromised spatial and axial resolutions and (2) warping and its induced aliasing and distortion. Future development of the platform will be aimed at mitigating these issues. For the hardware, (1) increase number of available microlenses in the field of view, (2) improve precision and reliability of the translation mechanism of cube inserts and sample/objective stage, and (3) design a vertical configuration and use mirrors to make the assembly more compact. To further improve the volumetric reconstruction, image post-processing to de-warp and de-alias may help. We could also complement LFM images with other imaging modalities (e.g. 2-photon or confocal microscopy) by image fusion using deep learning and further image post-processing.

\section{Supplementary Materials}

More detailed methodologies and CAD files can be found via the link below.

https://github.com/schultzlab/ultra-cheap-lightfield-microscope/

\section{Acknowledgments}

For the purpose of Open Access, the author has applied a CC BY public copyright license to any Author Accepted Manuscript (AAM) version arising from this submission.

The authors would like to acknowledge and appreciate support from MA Go, Y Liu, HJ Yoon, S $\mathrm{Lu}$, A Foust, H Isaac Verinaz-Jadan, J Page Vizcaino, P Fei, Z Wang.

\section{References}

[1] J. L. Fan et a, "High-speed volumetric two-photon fluorescence imaging of neurovascular dynamics," Nat. Commun. , vol. 11, 2020, https://doi.org/10.1038/s41467-020-19851-1

[2] M. Levoy, R. Ng, A. Adams, M. Footer, and M. Horowitz, "Light Field Microscopy," in $A C M$ SIGGRAPH 2006 Papers, 2006, pp. 924-934, doi: 10.1145/1179352.1141976.

[3] P. Quicke et al., "Single-neuron level one-photon voltage imaging with sparsely targeted genetically encoded voltage indicators," Front. Cell. Neurosci., vol. 13, no. February, pp. 1-12, 2019, doi: 10.3389/fncel.2019.00039.

[4] B. Diederich et al., "A versatile and customizable lowcost 3D-printed open standard for microscopic imaging," Nat. Commun., vol. 11, no. 1, 2020, doi: 10.1038/s41467-020-19447-9.

[5] D. Armendariz, "Aperture and F-number," Art Sci. Digit. Photogr., vol. CS194-23, pp. 4-7, [Online]. Available: https://inst.eecs.berkeley.edu/ cs19423/sp13/slides/ApertureAndFNumber.pdf.

[6] M. Broxton et al., "Wave optics theory and 3-D deconvolution for the light field microscope," Opt. Express, vol. 21, no. 21, p. 25418, 2013. doi: 10.1364/oe.21.025418.

[7] P. Quicke, "Improved Methods for Functional Neuronal Imaging with Genetically Encoded Voltage Indicators.," 2019. https://doi.org/10.25560/73910 